\title{
Damage Detection of Bridge Structure Based on SVM
}

\author{
Yaojin Bao, ${ }^{1}$ Chenjin Song, ${ }^{1}$ Wensi Wang, ${ }^{2}$ Ting Ye, ${ }^{2}$ Lu Wang, ${ }^{3}$ and Lan Yu ${ }^{4}$ \\ ${ }^{1}$ College of Civil Engineering, Beijing Jiaotong University, Beijing 100044, China \\ ${ }^{2}$ Transportation Management College, Dalian Maritime University, Dalian 116026, China \\ ${ }^{3}$ China Academy of Civil Aviation Science and Technology, Beijing 100028, China \\ ${ }^{4}$ Yanching Institute of Technology, Beijing 065201, China \\ Correspondence should be addressed to Yaojin Bao; bao_yaojin@163.com
}

Received 26 September 2013; Revised 17 October 2013; Accepted 17 October 2013

Academic Editor: Rui Mu

Copyright (C) 2013 Yaojin Bao et al. This is an open access article distributed under the Creative Commons Attribution License, which permits unrestricted use, distribution, and reproduction in any medium, provided the original work is properly cited.

For bridge management and maintenance, it is important to detect the damage of bridge pier. Due to the complexity of damage detection, an effective method is very interesting. Support vector machine (SVM) is used to detect the damage of bridge pier in this paper. To improve the detection accuracy of SVM, Grubbs' test method is adopted to delete the outliers for SVM. Then, a numerical analysis is used to determine the input parameters for SVM. Lastly, the comparison results between the proposed SVM and the actual measure value suggested that the proposed SVM is a powerful tool for detecting damage of bridge pier.

\section{Introduction}

Many factors, like damage on the railway, may lead to the instability of railway bridge. The pier is a main part of bridge structure and its safety has been an essential element for the bridge. The state of generalized pier which includes the bridge pier and its base affects the safety of the entire bridge, as the important part supporting the upper structure. The pier in bad condition would affect the function of the entire bridge even though the beam is in good condition. There will be various damages on the railway pier because it not only bears the dynamic load of the train but also suffers from some outside influences such as flood erosion, material erosion caused by the ground and the groundwater, and sudden earthquake. These damages would break the pier base, reduce the stiffness, make the pier lack of durability and bearing capacity, and cause other problems. It will affect the safety of the entire bridge. Particularly, it will be a severe damage when a train is running on the bridge. In recent years, in order to increase the train speed and provide heavyweight freight train running successfully, a complete investigation on the line is often performed to find the potential damage in time and meet the requirement of speed increase and higher heavyweight. Thus, it is necessary to provide an effective method to identify the safety condition of the pier.
The damage of the pier usually exists in the ground or under the water surface; it is difficult to judge the safety condition of the pier only with the appearance inspection. Since this type of damage is expected to simulate the condition in which a bridge suffers from nonuniform pier settlement or hidden damage inside piles of buried foundations, this kind of damage is introduced by cutting one of the bridge piers at the footing level. Therefore, it is general to take a dynamic response of the pier under train excitation as an index to assess the safety condition. If the value of the index is beyond the threshold, it suggests that there would be some dangers about the pier [1-5].

The basic principle of the dynamic response can be described as follows: the damage would bring about changes in stiffness, quality, and damping of the pier structure, which will change the dynamic characteristic parameters of the structure (e.g., natural frequency and mode shape). Therefore, the changing condition of the structure damage can be detected based on the change of the dynamic characteristic parameters. However, it will cost quite a long time and enormous expenses using this method. In addition, the above safety assessment model needs to take many elements into consideration; however, most assessment models only consider some specific parameters. Therefore, it is very important 
to develop a convenient and accurate safety assessment method for the pier.

In recent years, among all the damage detection methods of civil engineering structure, the damage detection method based on vibration modal analysis theory is the most widely used. The vibration modal analysis method involves many dynamic characteristic parameters such as modal frequency, modal type, curvature modal, and flexibility curvature. It is difficult to build the nonlinear relationship between the damage indicator and the structural breakage. This is because calculating the damage identification of modal structure is a complex mathematical problem.

Recently, support vector machine (SVM), which can be found in Vapnik [6-8], is a kind of statistical learning method which can achieve the best generalization effect by using a limit number of sample information to find the optimal compromise between the reasoning ability and the complexity of the model. The method has advantages in solving high-dimensional pattern recognition and nonlinear and small sample events and can be promoted and applied in regression analysis and other problems. Many studies show that SVM is an effective method to deal with systematical modeling and control of complex nonlinear problems [914]. It has also been proposed as a novel technique in detection problem, such as vehicle detection [15], trafficpattern recognition [16], and head recognition [17]. These successful applications motivate us to apply SVM in damage detection of bridge pier.

The damage detection model of bridge pier based on Grubbs' test method and SVM mainly aims at getting the relative information from the input data and other aspects to solve damage detection problem of bridge pier. Before the application of SVM, data preprocessing is necessary for SVM to eliminate the abnormal data from input data. However, individual data often deviates from the expectation or a large number of statistical values in simple data processing. The results may be not accurate if these abnormal data are deleted as the input data. That is, if these abnormal data are simply rejected, the operation may delete the hidden useful information indicated by these abnormal data. Therefore, how to determine and eliminate the abnormal values is a key issue in data processing. Grubbs' test method is a statistical test used to detect outliers in a univariate data set, which has been proved in some literatures $[18,19]$. Thus, Grubbs' test method is also used to detect outliers of SVM model.

To provide an effective method to detect bridge structure, this paper attempts to detect the damage of bridge pier based on Grubbs' test method and SVM. Therefore, this paper is organized as follows. Section 2 provides a brief introduction to the detected model based on Grubbs' test method and SVM; Section 3 presents numerical analysis and parameter selection. Section 4 reveals results of a numeral test and the prediction model. Lastly, the conclusions are stated in Section 5.

\section{The Damage Detection Model of Bridge Pier}

The damage detection model of bridge pier consists of two phases. One is the process of data processing in which

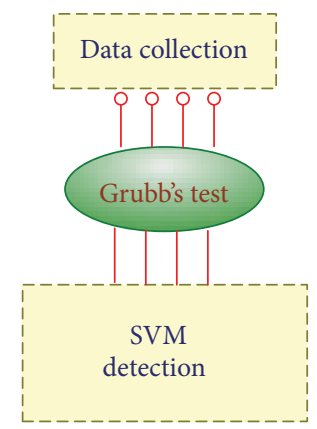

Figure 1: The structure of detection model.

Grubbs' test method is used and the other is the process of damage detection in which SVM is used. The model can be described as in Figure 1. Then, the two parts of the detection model are discussed, respectively.

2.1. Applying Grubbs' Test Method in Deleting Outliers. The abnormal data are processed based on Grubbs' criterion. It is greatly necessary to eliminate the abnormal data in data processing to improve the prediction accuracy. Therefore, the process of Grubbs' test method for deleting outliers is as follows. Firstly, the abnormal values usually would affect the prediction procedure; for example, there will be error codes if the abnormal values are added into the running code, so that the program cannot work normally. Secondly, the abnormal values usually would affect the prediction results. However, some undiscovered abnormal values may produce reasonable results under certain conditions. These abnormal values, if they have no regular variation and cannot affect the detection performance, are effective data samples and should be applied in the model rationally.

Grubbs' test method is usually used to detect outliers in small samples, where the number of outliers is unknown. Before the use of the method, all the data are assumed as normal distribution. In Grubbs' test method, only one abnormal value can be deleted every time. Thus, there are multiple eliminations for the data samples. Grubbs' test method will not come to an end until there are no abnormal values in the total samples. However, multistep iterative would affect the detection probability; the method is suitable for the samples with the number more than six.

Grubbs' test method is based on the following assumptions.

(H0) There is no abnormal value in the data set.

(Ha) There is at least one abnormal value in the data set.

Grubbs' test method can be described as follows:

$$
G=\frac{\max _{i=1, \ldots, n}\left|Y_{i}-\bar{Y}\right|}{g},
$$

where $\bar{Y}$ and $g$ represent the sample mean and the standard deviation, respectively. The statistic of Grubbs' test method is the deviation degree of the data with the maximum deviation compared with the standard value of unit sample. 
Equation (1) reflects a double-sided test. Grubbs' test method can also be a one-sided test. In order to test whether the minimum value in the sample is an abnormal one or not, the following equation can be used:

$$
G=\frac{\bar{Y}-Y_{\min }}{g},
$$

where $Y_{\min }$ represents the minimum value of the sample. In order to test whether the maximum value in the sample is an abnormal one or not, the following equation can be used:

$$
G=\frac{Y_{\max }-\bar{Y}}{g} .
$$

For the double-sided test, if the significance level $a$ satisfies the following conditions in (4), it means that there exist abnormal values in the sample:

$$
G>\frac{N-1}{\sqrt{N}} \sqrt{\frac{t_{a /(2 N), N-2}^{2}}{N-2+t_{a /(2 N), N-2}^{2}},}
$$

where $t_{a /(2 N), N-2}^{2}$ represents the upper threshold of $t$ distribution under the premise of $N-2$ degrees of freedom and significance level $a /(2 N) . a /(2 N)$ can be substituted for $a / N$ in the one-sided test.

2.2. The Basic Principle of SVM. Support vector machine is a learning linear machine which is based on the structural risk minimization (SRM) inductive principle, as opposed to the commonly used empirical risk minimization (ERM) approach. SRM attempts to minimize an upper bound of the generalization error which consists of the sum of the training error and a confidence level. ERM tries to minimize the training error. Therefore, SVM has a higher generalization performance.

Given a set of data points $\left(x_{1}, y_{1}\right),\left(x_{2}, y_{2}\right), \ldots,\left(x_{i}, y_{i}\right)$ $\left(x_{i} \in X \subseteq R^{n}, y_{i} \in Y \subseteq R\right.$ ), SVM can map $x_{i}$ to a highdimensional feature space $H$ using nonlinear mapping $\phi$ and conduct the linear approximation in this space, which can be described as follows:

$$
f(x)=\omega \cdot \phi(x)+b,
$$

where $\omega$ is the weight vector of the hyperplane $b$ is the bias term.

According to the statistical principles, the minimum and regular risk generic function can be attained under certain constraints to determine $\omega$ and $b$ :

$$
\begin{gathered}
\min \quad \frac{1}{2}\|\omega\|^{2}+C \frac{1}{l} \sum_{i=1}^{l}\left(\xi_{i}+\xi_{i}^{*}\right) \\
\text { s.t. } \quad y_{i}-\omega \cdot \varphi\left(x_{i}\right)-b \leq \varepsilon+\xi_{i} \\
\omega \cdot \phi\left(x_{i}\right)+b-y_{i} \leq \varepsilon+\xi_{i}^{*}, \quad i=1, \ldots, l \\
\xi_{i}^{*} \geq 0 .
\end{gathered}
$$

The first term in formula (6) is named as the regular one, which makes the function more flat to improve its generic abilities; the second term is the experience risk generic function, which can be determined by different loss functions, among which the constraint $C>0$ controls the punishment level for the samples beyond error $\varepsilon$. The meaning of formula (6) can be found in the literature [11].

Formula (6) is a convex quadratic optimization problem, which can be solved by introducing the Lagrange function, shown in the following equation:

$$
\omega-\sum_{i=1}^{l}\left(a_{i}-a_{i}^{*}\right) x_{i}=0 .
$$

Then,

$$
f(x)=\sum_{i=1}^{l}\left(a_{i}-a_{i}^{*}\right) \phi\left(x_{i}\right) \cdot \phi(x)+b .
$$

Let $K\left(x_{i}, x_{j}\right)=\phi\left(x_{i}\right) \cdot \phi(x)$; then,

$$
f(x)=\sum_{i=1}^{l}\left(a_{i}-a_{i}^{*}\right) K\left(x_{i}, x\right)+b,
$$

where $K\left(x_{i}, x_{j}\right)$ is the inner product of vectors $x_{i}$ and $x_{j}$ in feature spaces $\phi\left(x_{i}\right)$ and $\phi\left(x_{j}\right)$, which is named as the kernel function through which all the computations cannot be mapped to high-dimensional spaces but can be directly conducted in the input space. The kernel functions are the key parts of SVM; different SVMs consist of different kernel functions.

\section{The Numerical Analysis of Natural Vibration Characteristics of the Pier}

3.1. The FEM Analysis Model. The rectangular pier is made of reinforced concrete. The side soil mass of the concrete spread foundation is made of semidry and hard cohesive soil and the basal soil mass is made of pebble soil. The pier is 8 meters high and 3.6 meters and 1.6 meters width on average in the horizontal and longitudinal directions, respectively. The bottom 4 meters of the foundation is under the ground. The numerical analysis model of the pier is shown in Figure 2.

In order to simplify the calculation, some assumptions are made as follows.

(1) The pier body uses block units and the constraints imposed on the foundation by the soil mass are simulated through the linear spring.

(2) Ignoring the constraints imposed on the pier by the beam and the rail, the upper beam structure which is a one-hole beam including ballast, rail system, sidewalk panel, and other accessory structures is taken as the lumped mass to impose on the top of the pier.

3.2. The Computing Parameters of the Model. The constraints imposed on the pier by the soil mass include the transverse constraint for the side of the foundation and the longitudinal 


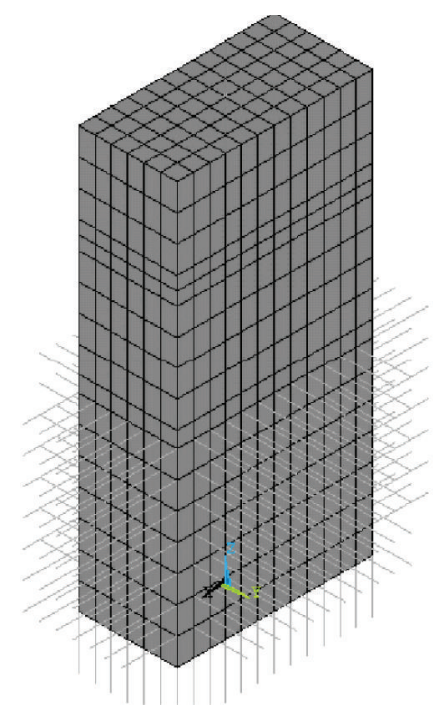

Figure 2: The FEM model of the fifth pier.

constraint for the bottom of the foundation, which are simulated through horizontal spring $k_{h}$ and vertical spring $k_{v}$. The spring coefficients based on the " $m$ " method can be acquired in the literature [20].

(1) The Coefficient Calculation of the Lateral and Horizontal Springs. The pier foundation is discretized into $n$ units and $h_{i}$ meters each unit, as shown in Figure 3. The restriction imposed on the base by the foundation soil is equivalent to the spring applied to each node of the unit. If the effective width of the lateral base is $b_{0}$, then the spring coefficient of the nodes in the lateral base can be attained as follows.

The spring coefficient $k_{x 1}^{\prime}$ of the lateral foundation soil within the range of unit (1) is the product of the triangle area and the unit width $b_{0}$ :

$$
k_{x 1}^{\prime}=b_{0}\left(\frac{m h_{1}}{2}\right) h_{1}=\frac{m b_{0} h_{1}^{2}}{2},
$$

where $m$ is the scale factor of the horizontal spring coefficients of the soil changing with the depth.

The lateral spring coefficients imposed on node 1 and node 2 of unit (1) shown in Figure 3 are determined based on the following equations:

$$
\begin{aligned}
& k_{x 11}^{\prime}=\frac{k_{x 1}^{\prime}}{3}, \\
& k_{x 12}^{\prime}=\frac{2 k_{x 1}^{\prime}}{3} .
\end{aligned}
$$

In the same way, the lateral spring coefficients of other element nodes are as follows:

$$
k_{x n}^{\prime}=\frac{b_{0} m\left[2 *\left(h_{1}+\cdots+h_{n-1}\right)+h_{n}\right] h_{n}}{2} .
$$

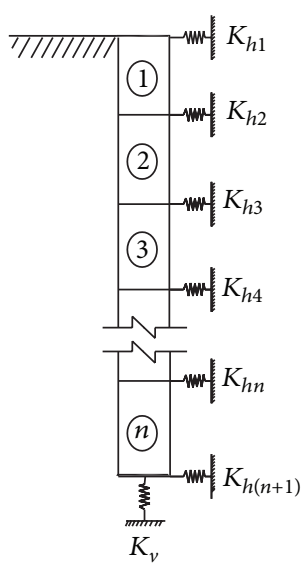

(a)

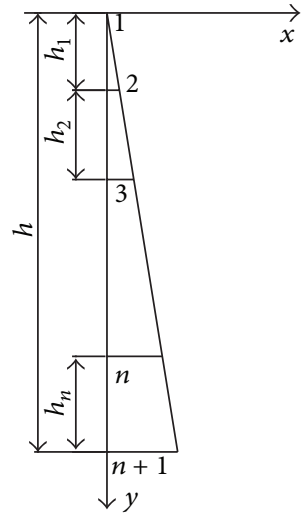

(b)
Figure 3: The calculation model of elastic coefficients.

Then,

$$
\begin{aligned}
k_{x n n}^{\prime} & =\frac{k_{x n}^{\prime}}{3}, \\
k_{x n(n+1)}^{\prime} & =\frac{2 k_{x n}^{\prime}}{3} .
\end{aligned}
$$

Based on the above analysis, the equivalent spring coefficients of each node can be attained as follows:

$$
\begin{gathered}
K_{h 1}=k_{x 11}^{\prime} \\
K_{h 2}=k_{x 12}^{\prime}+k_{x 22}^{\prime} \\
\vdots \\
K_{h n}=k_{x(n-1) n}^{\prime}+k_{x n n}^{\prime} \\
K_{h(n+1)}=F_{x n(n+1)} .
\end{gathered}
$$

(2) The Calculation of the Vertical Spring Coefficient of the Base Ground. The underside rotation of the pier base around the vertical plane can be considered, ignoring the horizontal vibration. The vertical spring coefficient of the underside of the base ground is calculated as follows:

$$
k_{v}=C_{0} A_{0},
$$

where $C_{0}$ is the vertical foundation coefficient of the base bottom and $A_{0}$ is the stressed area of the base bottom.

(3) The Constraint Simulation in the State of Foundation Scouring. According to the literature [21], one meter depth of soil suffering from foundation scouring is taken as an example; the earth pressure in the scouring state is shown in Figure 4 in which $W_{2}, W_{3}, \ldots, W_{n}$ represent the weight of each layer of soil mass.

The influence of soil scouring of the base side on the base constraint is embodied in two aspects:

(a) after the soil scouring in the side of the base, the horizontal constraint of this layer of soil on the base can be deleted; 


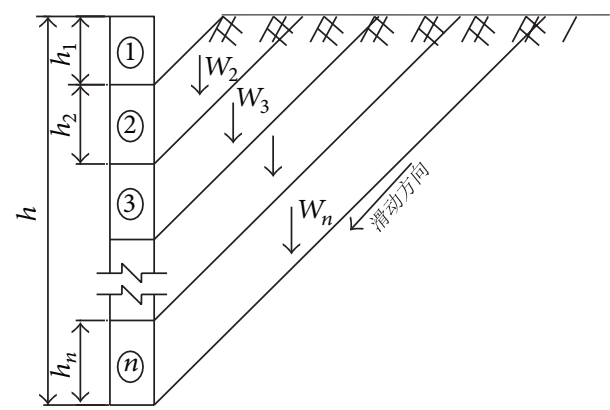

FIGURE 4: The distribution of soil pressure on the sliding surfaces.

TABLE 1: The calculation results of natural vibration frequency of the pier.

\begin{tabular}{lcccc}
\hline $\begin{array}{l}\text { Working } \\
\text { condition }\end{array}$ & $\begin{array}{c}\text { Foundation } \\
\text { depth }(\mathrm{m})\end{array}$ & $\begin{array}{c}\text { Frequency } \\
(\mathrm{Hz})\end{array}$ & $\begin{array}{c}\text { Frequency } \\
\text { reduction } \\
(\mathrm{Hz})\end{array}$ & $\begin{array}{c}\text { Relative } \\
\text { frequency } \\
\text { reduction }(\mathrm{Hz})\end{array}$ \\
\hline Original state & 4.0 & 7.02 & $/$ & $/$ \\
Digging $1 \mathrm{~m}$ & 3.0 & 6.67 & 0.34 & 0.34 \\
Digging $2 \mathrm{~m}$ & 2.0 & 6.45 & 0.57 & 0.23 \\
Digging $3 \mathrm{~m}$ & 1.0 & 6.31 & 0.71 & 0.14 \\
\hline
\end{tabular}

(b) the lateral spring coefficient of the next layer of soil would change due to the soil scouring in this layer of soil.

From Figure 4, it can be found that it will have less influence on the lateral coefficient of the soil in the next layer if the side soil mass of the base is not much reduced because the soil is dug within a small range. The lateral spring coefficient of the base under the scoured region can still be calculated based on (13)-(17).

The lateral spring coefficients of the base are computed according to (19); the lateral spring coefficients with other scouring depth are calculated in the same way:

$$
\begin{gathered}
k_{x 1}^{\prime}=0, \\
k_{x 2}^{\prime}=\frac{b_{0}\left(2 m h_{1}+h_{2}\right) h_{2}}{2}, \\
K_{h 1}=0, \\
K_{h 2}=k_{x 12}^{\prime}+k_{x 22}^{\prime}=0+k_{x 22}^{\prime}, \\
K_{h 3}=k_{x 23}^{\prime} .
\end{gathered}
$$

There will be less influence on the vertical coefficients of the base bottom if the side soil mass of the base is not much reduced, neglecting the change of the vertical spring coefficients of the base bottom caused by the scouring.

3.3. The Numerical Analysis Results. The natural vibration characteristics of the fifth pier in the original state and under different digging depths and the natural vibration frequency
TABLE 2: The amplitude ratio of the pier.

\begin{tabular}{lccc}
\hline Working condition & Pier vertex & Pier midpoint & Pier subpoint \\
\hline Original state & 2.04 & 1.52 & 1 \\
Digging $1 \mathrm{~m}$ & 2.03 & 1.51 & 1 \\
Digging $2 \mathrm{~m}$ & 2.02 & 1.50 & 1 \\
Digging $3 \mathrm{~m}$ & 2.01 & 1.49 & 1 \\
\hline
\end{tabular}

are shown in Table 1 . Table 2 shows the amplitude ratio of the pier under four kinds of digging states.

\section{Case Studies}

4.1. The Parameters for SVM. In fact, when SVM is training, the input parameters with enough high accuracy, the damage detection results will be accurate and effective. From the numerical analysis results, it can be found that the natural vibration frequency in different positions shows different results. Therefore, it is reasonable to choose depth, working condition, and natural vibration frequency as the input parameters for SVM model.

Before the application of SVM model, the data should be normalized firstly. Then, the normalized data will be divided into three parts of sets, which will be used for the training, testing, and inspection, respectively. That is, there are about $70 \%$ samples for training, $10 \%$ samples for testing, and the remaining samples for inspection. Thus, negative root mean squared error (NRMSE) is also adopted to reflect the prediction accuracy in this paper:

$$
\text { fit }=-\left[\frac{\sum_{i=1}^{n}(V-\widehat{V})}{n-p}\right]^{1 / 2},
$$

where $\widehat{V}$ is the prediction value by the model, $V$ is the observed value, $n$ is the number of observations, and $p$ is the number of model parameters which refers to the literature [22].

The performance of SVM mainly referred to the ability of classifying unknown data samples correctly (i.e. the generalization ability). The kernel function is the key part for SVM. It is because the kernel function provides a simple bridge from linearity to nonlinearity for SVM. In general, the RBF kernel, as a nonlinearly kernel function, is a reasonable first choice [23]. There are three parameters while using RBF kernels: $C, \varepsilon$, and $\gamma$. The parameter selection is important for the performance of the algorithm. However, it is not known a priori which of $C, \varepsilon$, and $\gamma$ is the best choice for the problem. To properly select the three parameters, there are several methods adopted to identify the best parameter value. Among these methods, grid search is frequently used. Therefore, the grid search is also used to determine the values of parameters $(C, \varepsilon$, and $\gamma)$. At last, the three parameters are selected as 3.5423, 0.0064, and 1.0698 .

4.2. The Detection Results Based on SVM. The damage degree of the pier structure was assumed as $3.05 \%, 6.15 \%, 11.5 \%$, and $14.5 \%$, respectively. After the samples have been trained 
TABLE 3: The comparison results between the SVM and actual observation.

\begin{tabular}{lccc}
\hline $\begin{array}{l}\text { Working } \\
\text { condition }\end{array}$ & $\begin{array}{c}\text { Foundation } \\
\text { depth }(\mathrm{m})\end{array}$ & Results of SVM & Actual results \\
\hline Original state & 4.0 & $14.5 \%$ & $14.64 \%$ \\
Digging 1 m & 3.0 & $11.5 \%$ & $11.61 \%$ \\
Digging 2 m & 2.0 & $6.15 \%$ & $6.21 \%$ \\
Digging 3 m & 1.0 & $3.05 \%$ & $3.10 \%$ \\
\hline
\end{tabular}

in data processing by Grubbs' test method, the damage data with $20 \%$ were taken as the testing samples in this paper. The results of SVM can be seen in Table 3.

From the comparison results in Table 3, it can be found that the results of the proposed SVM are near to the measured values. This indicates that the proposed SVM is effective for damage detection of bridge structure.

\section{Conclusions}

For bridge management and maintenance, it is an important task to evaluate the working state of existing railway piers. It is also an important measure to ensure the safety of trains with increased speed or heavy loads. However, it is hard to detect the damage of bridge pier because the pier is under the water or there are lots of factors affecting the damage. SVM, as a learning machine, does not need a specific function and can reflect the relationship in nonlinear and real-time system of input and performance. Therefore, this paper attempted to use SVM to detect the damage of bridge pier. Considering that the data processing is important for the performance of SVM, Grubbs' test method is adopted to delete outliers for SVM. Since there are lots of factors which affect the damage of pier, a numerical analysis is used to determine the input parameters for SVM. The proposed SVM detection model was tested on the data from field experiments. The results show that the model has a good performance for detecting damage of bridge pier, by comparing with that of the measure values. Thus, the detection model is proved to be a powerful tool for detecting damage of bridge pier.

\section{Acknowledgments}

The research is sponsored by the Major State Basic Research Development Program of China (“973" Program: 2013CB036203), the Program for New Century Excellent Talents in University (NCET-10-0219) and Fundamental Research Funds for the Central Universities 3132013079.

\section{References}

[1] X. Luo, H. Haya, T. Inaba, T. Shiotani, and Y. Nakanishi, "Damage evaluation of railway structures by using train-induced AE," Construction and Building Materials, vol. 18, no. 3, pp. 215-223, 2004.

[2] H. Xia, N. Zhang, and G. De Roeck, "Dynamic analysis of high speed railway bridge under articulated trains," Computers and Structures, vol. 81, no. 26-27, pp. 2467-2478, 2003.
[3] H. Xia, N. Zhang, and R. Gao, "Experimental analysis of railway bridge under high-speed trains," Journal of Sound and Vibration, vol. 282, no. 1-2, pp. 517-528, 2005.

[4] H. Xia, G. De Roeck, N. Zhang, and J. Maeck, "Experimental analysis of a high-speed railway bridge under Thalys trains," Journal of Sound and Vibration, vol. 268, no. 1, pp. 103-113, 2003.

[5] D. M. Siringoringo, Y. Fujino, and T. Nagayama, "Dynamic characteristics of an overpass bridge in a full-scale destructive test," Journal of Engineering Mechanics-ASCE, vol. 139, no. 6, pp. 691-701, 2013.

[6] V. N. Vapnik, The Nature of Statistical Learning Theory, Springer, New York, NY, USA, 1995.

[7] V. N. Vapnik, "An overview of statistical learning theory," IEEE Transactions on Neural Networks, vol. 10, no. 5, pp. 988-999, 1999.

[8] V. N. Vapnik, The Nature of Statistical Learning Theory, Statistics for Engineering and Information Science, Springer, New York, NY, USA, 2nd edition, 2000.

[9] L. J. Cao and F. E. H. Tay, "Support vector machine with adaptive parameters in financial time series forecasting," IEEE Transactions on Neural Networks, vol. 14, no. 6, pp. 1506-1518, 2003.

[10] B. Z. Yao, C. Y. Yang, J. B. Yao, and J. Sun, “Tunnel surrounding rock displacement prediction using support vector machine," International Journal of Computational Intelligence Systems, vol. 3, no. 6, pp. 843-852, 2010.

[11] Y. Bin, Y. Zhongzhen, and Y. Baozhen, "Bus arrival time prediction using support vector machines," Journal of Intelligent Transportation Systems, vol. 10, no. 4, pp. 151-158, 2006.

[12] B. Yu, Z. Z. Yang, K. Chen, and B. Yu, "Hybrid model for prediction of bus arrival times at next station," Journal of Advanced Transportation, vol. 44, no. 3, pp. 193-204, 2010.

[13] B. Yu, W. H. K. Lam, and M. L. Tam, "Bus arrival time prediction at bus stop with multiple routes," Transportation Research Part C, vol. 19, no. 6, pp. 1157-1170, 2011.

[14] B. Yu, Z. Z. Yang, and S. Li, "Real-time partway deadheading strategy based on transit service reliability assessment," Transportation Research Part A, vol. 46, no. 8, pp. 1265-1279, 2012.

[15] Z. Sun, G. Bebis, and R. Miller, "Improving the performance of on-road vehicle detection by combining Gabor and wavelet features," in Proceeding of the IEEE 5th International Conference Intelligent Transportation Systems, pp. 130-135, 2002.

[16] J. T. Ren, X. L. Ou, Y. Zhang, and D. C. Hu, "Research on network-level traffic pattern recognition," in Proceeding of the IEEE 5th International Conference Intelligent Transportation Systems, pp. 500-504, 2002.

[17] R. Reyna, A. Giralt, and D. Esteve, "Head detection inside vehicles with a modified SVM for safer airbags," in Proceeding of the IEEE Intelligent Transportation Systems, pp. 268-272, Oakland, Calif, USA, August 2001.

[18] M. S. Srivastava, "Effect of equicorrelation in detecting a spurious observation," The Canadian Journal of Statistics, vol. 8, no. 2, pp. 249-251, 1980.

[19] J. K. Baksalary and S. Puntanen, "A complete solution to the problem of robustness of Grubbs's test," The Canadian Journal of Statistics, vol. 18, no. 3, pp. 285-287, 1990.

[20] W. M. Cai and Z. W. Hu, Soil Mechanics and Foundation Engineering, China Building Industry Press, Beijing, China, 1991 Chinese.

[21] A. Nishimura, "Examination of bridge substructure for integrity," Japanese Railway Engineering, no. 114, pp. 13-17, 1990. 
[22] B. Dong, C. Cao, and S. E. Lee, "Applying support vector machines to predict building energy consumption in tropical region," Energy and Buildings, vol. 37, no. 5, pp. 545-553, 2005.

[23] S. S. Keerthi and C. J. Lin, "Asymptotic behaviors of support vector machines with gaussian kernel," Neural Computation, vol. 15, no. 7, pp. 1667-1689, 2003. 


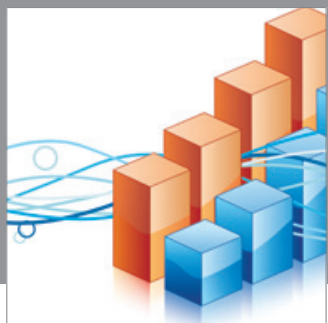

Advances in

Operations Research

mansans

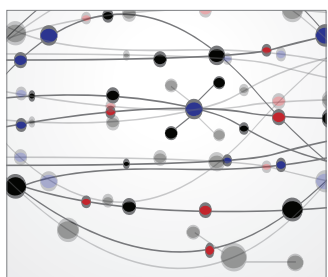

The Scientific World Journal
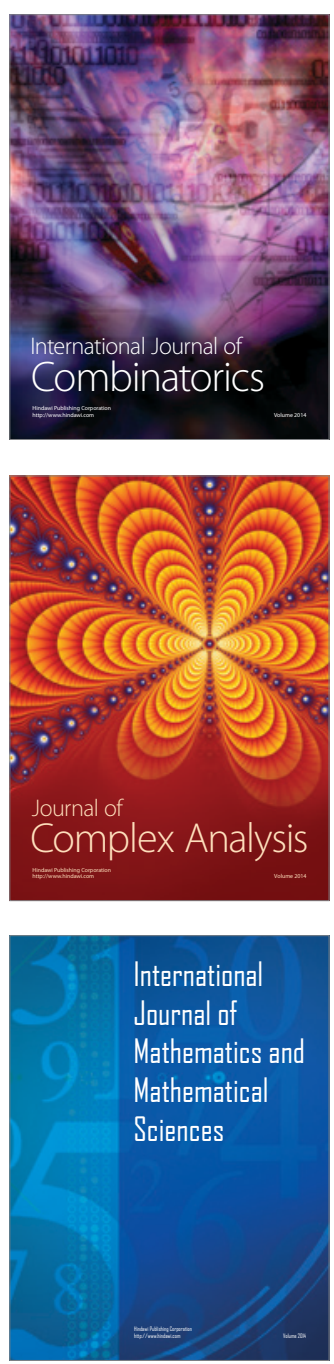
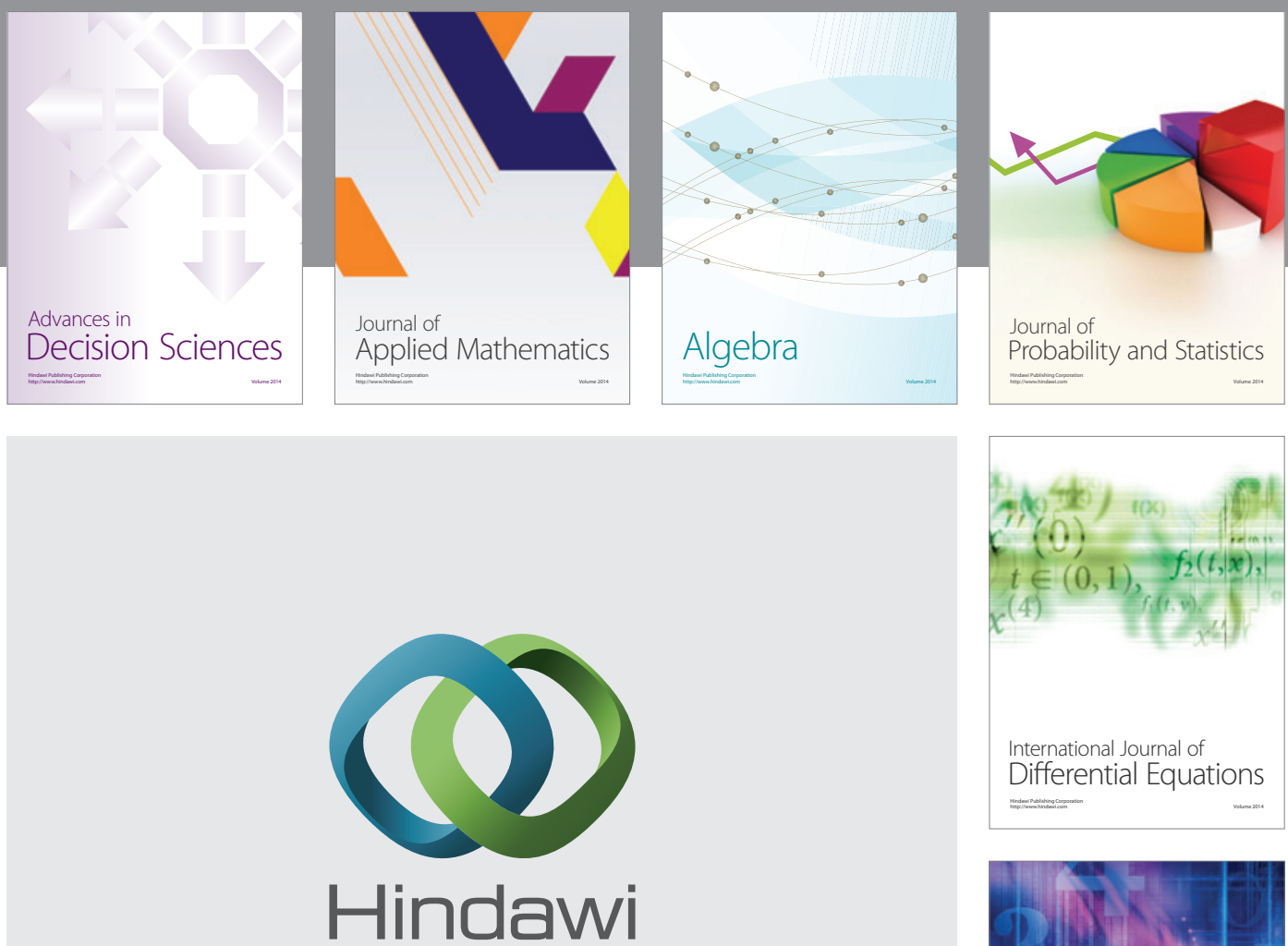

Submit your manuscripts at http://www.hindawi.com
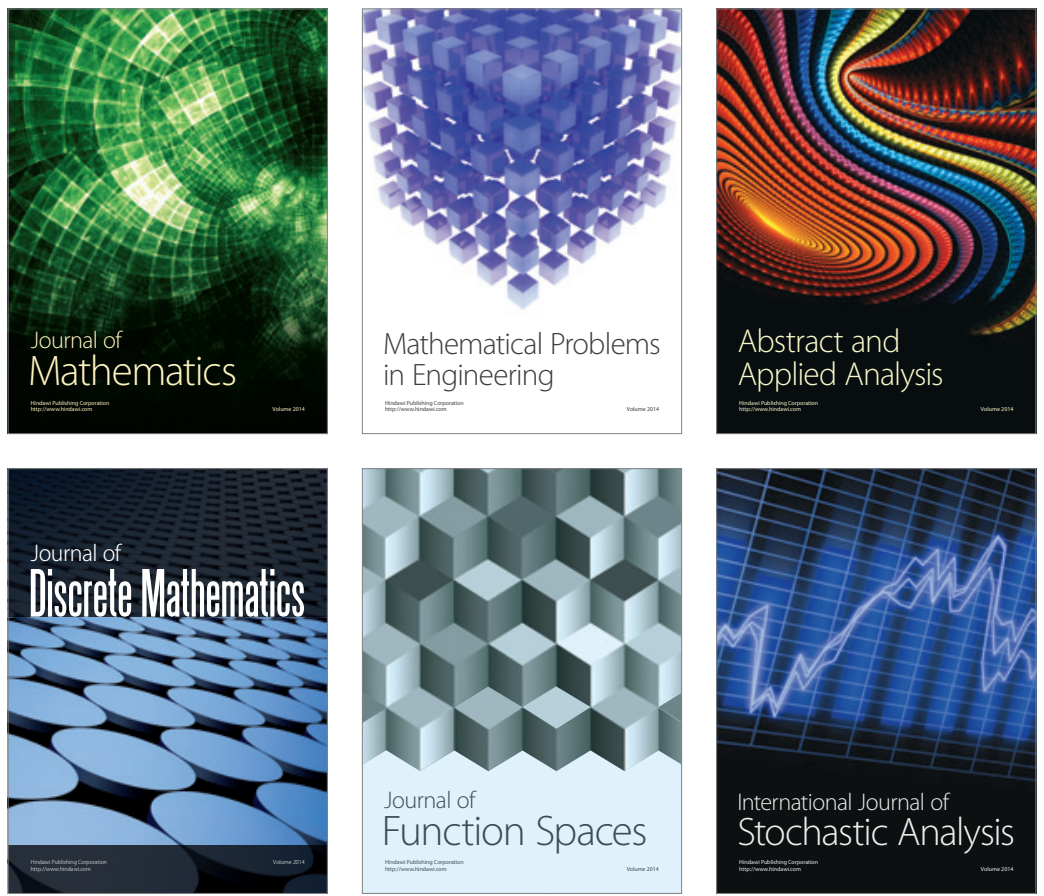

Journal of

Function Spaces

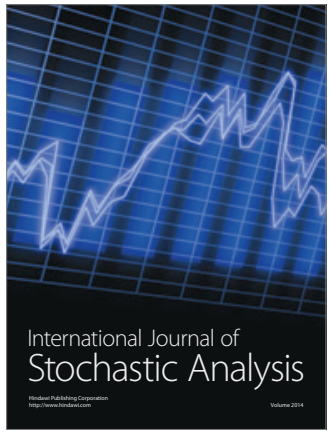

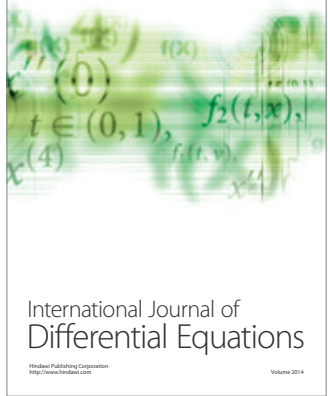
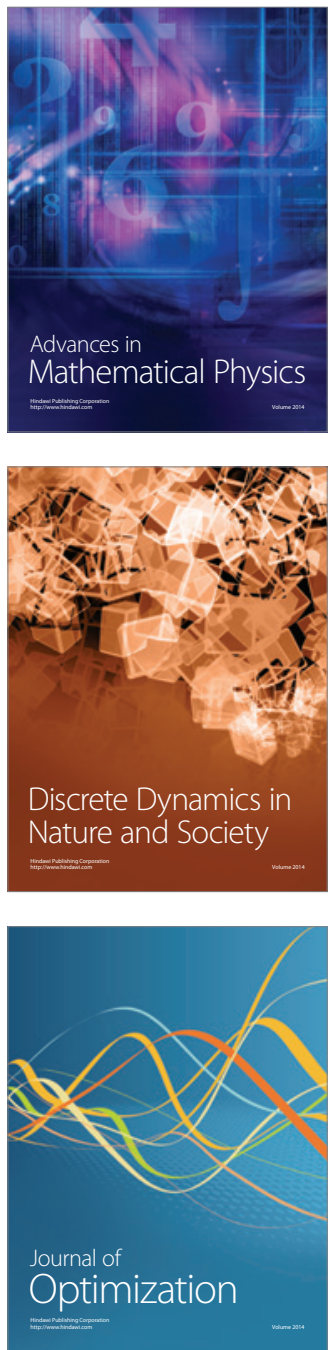